\title{
Wenning, Norbert
}

\section{Differenz durch Normalisierung}

formal überarbeitete Version der Originalveröffentlichung in: formally revised edition of the original source in:

Lutz, Helma [Hrsg.]; Wenning, Norbert [Hrsg.]: Unterschiedlich verschieden. Differenz in der Erziehungswissenschaft. Opladen : Leske + Budrich 2001, S. 275-295

Bitte verwenden Sie in der Quellenangabe folgende URN oder DOI /

Please use the following URN or DOI for reference:

urn:nbn:de:0111-opus-26229

10.25656/01:2622

https://nbn-resolving.org/urn:nbn:de:0111-opus-26229

https://doi.org/10.25656/01:2622

\section{Nutzungsbedingungen}

Gewährt wird ein nicht exklusives, nicht übertragbares, persönliches und beschränktes Recht auf Nutzung dieses Dokuments. Dieses Dokument ist ausschließlich für den persönlichen, nicht-kommerziellen Gebrauch bestimmt. Die Nutzung stellt keine Übertragung des Eigentumsrechts an diesem Dokument dar und gilt vorbehaltlich der folgenden Einschränkungen: Auf sämtlichen Kopien dieses Dokuments müssen alle Urheberrechtshinweise und sonstigen Hinweise auf gesetzlichen Schutz beibehalten werden. Sie dürfen dieses Dokument nicht in irgendeiner Weise abändern, noch dürfen Sie dieses Dokument für öffentliche oder kommerzielle Zwecke vervielfältigen, öffentlich ausstellen, aufführen, vertreiben oder anderweitig nutzen.

Mit der Verwendung dieses Dokuments erkennen Sie die Nutzungsbedingungen an.

\section{Terms of use}

We grant a non-exclusive, non-transferable, individual and limited right to using this document

This document is solely intended for your personal, non-commercial use. Use of this document does not include any transfer of property rights and it is conditional to the following limitations: All of the copies of this documents must retain all copyright information and other information regarding legal protection. You are not allowed to alter this document in any way, to copy it for public or commercial purposes, to exhibit the document in public, to perform, distribute or otherwise use the document in public.

By using this particular document, you accept the above-stated conditions of use.

\section{Kontakt / Contact:}

\section{peDOCS}

DIPF | Leibniz-Institut für Bildungsforschung und Bildungsinformation Informationszentrum (IZ) Bildung

E-Mail:pedocs@dipf.de

Internet: www.pedocs.de

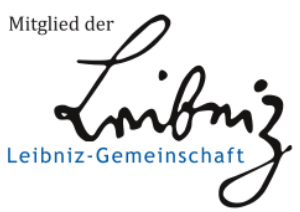


Helma Lutz/Norbert Wenning (Hrsg.)

\section{Unterschiedlich verschieden \\ Differenz in der Erziehungswissenschaft}

Leske + Budrich, Opladen 2001 
Die erste Auflage dieses Band erschien 2001 im Verlag Leske + Budrich, Opladen (jetzt VS-Verlag, Wiesbaden) und hatte folgende ISBN:

ISBN 3-8100-2854-1

Diese Version des Textes wird von der Herausgeberin und dem Herausgeber verantwortet. Sie macht den Gesamttext mit allen Beiträgen des genannten Bandes durch eine Open-Access-Publikation frei zugänglich. Das Werk ist dennoch insgesamt und in seinen einzelnen Teilen urheberrechtlich geschützt. Das Gesamtwerk bzw. die Einzelbeiträge dürfen nur mit Zustimmung der jeweiligen Autorinnen bzw. Autoren vervielfältigt, übersetzt oder in anderer Weise außerhalb der Grenzen des Urheberrechts genutzt werden. Jede kommerzielle Nutzung ist ausgeschlossen. 


\section{Inhaltsverzeichnis}

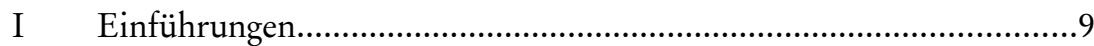

Differenzen über Differenz - Einführung in die Debatten.........................11

Helma Lutz, Norbert Wenning

Die Verwandlung der Philosophie in eine historische Diagnostik

der Differenzen.......................................................................................25

Rita Casale

Differenz und Differenzierung in soziologischer Perspektive

Frank Hillebrandt

Aspekte der angloamerikanischen pädagogischen Differenzdebatte:

Überlegungen zur Kontextualisierung.

Karin Amos

Egalitäre Differenz in der Bildung.

Annedore Prengel

II Disziplintheoretische Zugänge zu Differenz

Feministische Perspektiven auf „Differenz“ in Erziehungsund Bildungsprozessen.

Martina Löw

Das Soziale und die Differenz. Zur (De-)Thematisierung von

Differenz in der Sozialpädagogik.

Susanne Maurer 
Die Rezeption von Differenzdiskussionen in der Vergleichenden Erziehungswissenschaft

Karin Amos

Differenz als Konstitutionsproblem der Sonderpädagogik

Günther Opp, Michael Fingerle, Kirsten Pubr

III Kategorien zur Konstruktion von Differenz. .177

Frauen/Männer, Kinder/Erwachsene.

Rolf Nemitz

Kultur als Differenzierungskategorie.

Thomas Höhne

Differenz als Rechenaufgabe: über die Relevanz der Kategorien

Race, Class und Gender.....

Helma Lutz

An-, Zu- und Ungehörigkeiten Jugendlicher: Herkunft als Auskunft?....231 Clemens Dannenbeck, Hans Lösch, Felicitas Eßer

IV Zur Produktion von Differenz

Dichotome Differenzen und antirassistische Praxis. .251

Rudolf Leiprecht, Susanne Lang

Differenz durch Normalisierung. .275

Norbert Wenning

Die Autorinnen und Autoren. 


\section{Danksagung}

Dieses Buch ist nur zustande gekommen durch die Mitarbeit vieler HelferInnen mit unterschiedlichsten Beiträgen.

Wir bedanken uns insbesondere bei Ingrid Gogolin, Marianne KrügerPotratz, Rudolf Leiprecht, Karl-Ernst Ackermann und den Studierenden des Seminars „Differenzdebatten in der Erziehungswissenschaft“, das im Wintersemester 1999/2000 an der Westfälischen Wilhelms-Universität Münster stattfand, für interessante Hinweise und Kommentare zur inhaltlichen Gestaltung des Buches. Die Autorinnen und Autoren hatten sich mit vielfachen Änderungswünschen unsererseits zu befassen und dabei Ausdauer zu zeigen. Ulrike Fromm, Jeannette Stiller und Bernhard Rosenkötter haben mit viel Geduld die Korrekturen der diversen Versionen des Buches vorgenommen und umgesetzt. Dafür ein besonderes Dankeschön.

Wir hoffen, dass dieses Buch seinen Leserinnen und Lesern Anlass gibt zu heftigen Diskussionen, zu Revisionen und Perspektivwechseln, vielleicht auch zu Widerspruch.

Münster, Sommer 2000

Helma Lutz und Norbert Wenning

Hinweise zur Wiederauflage:

Die erneute Auflage dieses Bandes erfolgt, weil die Verlagsfassung im Buchhandel vergriffen ist und zugleich wiederholt Nachfragen nach dem Text an uns gerichtet werden. Eine vollständige Überarbeitung der Beiträge wäre nach fast zehn Jahren reizvoll, erscheint uns aber als recht aufwändig. Zudem hat sich die Debatte um Differenz in der Zwischenzeit weniger dynamisch entwickelt, als wir es erwartet bzw. erhofft hatten. Darum halten wir die Beiträge dieses Bandes auch in der vorliegenden Form grundsätzlich noch für aussagekräftig. Aus diesem Grund wählen wir eine „Zwischenlösung" und machen die Texte allgemein zugänglich. Die vorliegenden Texte sind seitenidentisch mit denen der ersten Auflage.

Da diese Fassung mit der Schrift UWR-GaramondNo8, die Verlagsfassung aber mit Garamond gesetzt ist, kommt es in manchen Absätzen zu leichten Verschiebungen.

Wir danken allen Beitragenden für ihre Zustimmung zu dieser Form der Veröffentlichung.

Frankfurt am Main, Landau in der Pfalz, Januar 2010

Helma Lutz und Norbert Wenning 


\title{
Differenz durch Normalisierung
}

\author{
Norbert Wenning
}

Mehrere Beiträge in diesem Band greifen in ihrer Argumentation u. a. auf den Begriff „Normalisierung“ zurück, ${ }^{1}$ der insbesondere durch die Werke Foucaults bekannt wurde. Hier geht es um die Frage, welche Wirkungen implizite (und explizite) Normalitätsvorstellungen in der Erziehungswissenschaft auf den Umgang mit bzw. auf die Produktion von Gleichheit und Differenz haben. Da Normalisierung als Begriff inzwischen relativ weit verbreitet ist, hat er unterschiedliche Bedeutungen und, je nach Kontext, abweichende Konnotationen (1.). Um diese zu verstehen, lohnt ein Blick in die Entstehungsgeschichte heute wirksamer Vorstellungen von Normalität (2.). Am Beispiel von Rousseaus Émile wird angedeutet, wie (überholte) Normalitätsvorstellungen in pädagogischen Theorien auch heute (noch) unbedacht etwa auf das pädagogische Menschenbild wirken können (3.). Darüber hinaus kann man z. B. Aspekte der jüngeren Subdisziplinbildung der Erziehungswissenschaft als Resultat der normalisierenden Wirkung pädagogischer Theorien begreifen (4.). Da andere Beiträge dieses Bandes Feministische Pädagogik und Sonderpädagogik ausführlich aufgreifen, steht hier Interkulturelle Erziehungswissenschaft bei der Frage nach Berechtigung und Wirkungen dieser subdisziplinären Aufgliederungen im Mittelpunkt. Anhand einer Diskussion aus einem Teilbereich der Interkulturellen Erziehungswissenschaft, der Frage der Ethnisierung durch ethnisch orientierte wissenschaftliche Erklärungsansätze, wird angedeutet, dass Normalisierungen im Bildungswesen mehr als rein akademische Folgen haben (5.). Insgesamt zeigt die Argumentation - wenn an dieser Stelle auch nur skizzenhaft -, dass Normalisierungsprozesse auch in der Erziehungswissenschaft durch die Verbreitung bestimmter Normalitätsannahmen Differenz bzw. Differenzvorstellungen erzeugen bzw. verstärken.

Explizit vor allem die Beiträge von Mauerer zur Sozialpädagogik, von Amos zur Vergleichenden Erziehungswissenschaft und von Opp, Fingerle und Puhr zur Sonderpädagogik. 


\section{Die Normalität der Normalisierung}

Normalisierung ist in politischen wie wissenschaftlichen Diskussionen ein seit längerer Zeit gebrauchter Begriff. In diesem Abschnitt geht es darum, aufzuzeigen, dass Normalisierung einerseits in der öffentlichen Auseinandersetzung weiter verbreitet ist, als etwa die jüngeren deutschen politischen bzw. historischen Diskussionen nach der politischen Wende von 1989/90 vermuten lassen. Normalisierung ist andererseits in vielen wissenschaftlichen Bereichen und (sozialen) Arbeitszusammenhängen eine unhinterfragte „diskurstragende Kategorie“ (Link 1997, S. 15). ${ }^{2}$

Mit der öffentlichen, vor allem politischen Verwendung von „Normalisierung" verbinden sich sowohl Hoffnungen als auch Befürchtungen. Die politische Benutzung zeigt dies deutlich: Wird zu Beginn der 1970er Jahre mit der Normalisierung der deutsch-deutschen Beziehungen die Anerkennung einer politischen Situation, vor allem der Wunsch nach politischer Entspannung und der Entwicklung hin zu „normalen“ Beziehungen verbunden, so speiste sich die nach der deutsch-deutschen Vereinigung 1990 aufkommende Diskussion aus einer anderen Quelle - dem Wunsch nach „Rückkehr“ bzw. nach Entwicklung hin zu einem normalen (National-) Staat. Den Kern der Diskussion bilden unterschiedliche Einschätzungen des Nationalstaats (Glotz 1994, Schoch 1996). Von konservativer Seite wurde 1990 die Rückbesinnung auf die wiedervereinigte Nation als Chance gesehen, die „vergangenheitsfixierte Identitätsneurose zu überwinden und ein normaler Nationalstaat in Europa zu werden" (Jarausch 1995, S. 572).

Hinter Normalisierung kann sich, mindestens für einen Teil der Betroffenen, Negatives verbergen. Im politisch rechten Lager wird z. B. mit bestimmten biopolitischen Vorstellungen Normalisierung im Sinne einer veränderten Normsetzung verbunden (Sierck 1995). Auch die Niederschlagung des Prager Frühlings in der Tschechoslowakei fand unter dem Stichwort Normalisierung statt. In diesem Fall heißt Normalisierung Repression und Abschließung. Derartige Verwendungen von Normalisierung unterstellen, es gäbe einen Zustand, der „normal“ im Sinne von allgemein positiv anerkannt bzw. früher schon einmal da gewesen sei. Damit sind politische Normalisierungen zumeist Beschreibungen von Veränderungsprozessen, die auf die (rückwärts gewandte) Anpassung an allgemein anerkannte $\mathrm{Zu}$ stände bzw. an solche, die diesen Anspruch erheben (können), abzielen.

Auch die wissenschaftliche Diskussion benutzt „Normalisierung“. Zwar fehlt dieser Terminus bisher in einschlägigen Handwörterbüchern, den-noch taucht er in verschiedenen, vor allem sozialwissenschaftlichen $\mathrm{Zu}$ sammenhängen auf, wie nachfolgend verdeutlicht wird. Der Begriff ist we-

Eine sehr breite und über Fachgrenzen hinweg reichende Darstellung von Normalisierungsprozessen liefert Jürgen Links „Versuch über den Normalismus“ (1997) mit dem bezeichnenden Untertitel „Wie Normalität produziert wird“. 
der neu noch in seiner Zuordnung zu einer Wissenschaft (bzw. Praxis) eindeutig. Zudem hat Normalisierung dabei unterschiedliche Bedeutungen und, je nach Kontext, abweichende Konnotationen.

In der Linguistik ist Normalisierung ein Fachbegriff: Er bezeichnet die gesellschaftliche Durchsetzung und Annahme einer (nationalen) Sprache auf allen gesellschaftlichen und funktionalen Ebenen der Kommunikation. Geht dies mit einer Normativierung, d. h. mit der Ausarbeitung referentieller sprachlicher Formen und Normen durch Grammatik, Lexikon und Graphieregeln, einher, kann es zur Entwicklung einer Standardsprache in einer Sprachgemeinschaft kommen (Kremnitz 1997, S. 26). Ein aktuelles Stichwort ist hier die letzte deutsche Rechtschreibreform.

In der EDV hat Normalisierung für die Erstellung von Datenbanken eine ähnliche Bedeutung wie in der Linguistik: als Versuch der Standardisierung. Andere Verwendungen sind Diskussionen um die Normalisierung des Drogengebrauchs bzw. -missbrauchs, also die Veränderung der Norm im Umgang mit Rauschmitteln. Im Strafvollzug wurde von Normalisierung gesprochen, d. h. von der Veränderung der Bedingungen des Freiheitsentzugs in Richtung eines „normalen“ Lebens. Das Normalisierungsprinzip als Ziel der Veränderung von Lebensbedingungen für Menschen mit geistiger Behinderung (z. B. Beck/Düe/Wieland 1996, Schildmann 1997, Gröschke 2000) zielt - überspitzt gesagt - auf die möglichst weitgehende Anpassung dieser Personen an eine als gegeben angesehene Normalität von „Leben“. In der Jugendarbeit dient der Terminus „Normalisierungsmuster" als Beschreibung eines Deutungsmusters in der Auseinandersetzung von Sozialarbeitern mit nichtdeutschen Jugendlichen. Oft wird betont, diese Jugendlichen seien genauso wie alle anderen, was von diesen häufig mit Anpassungen an die auf sie gerichteten Normalitätserwartungen erkauft ist (Auernheimer 1995, S. 238). Im Zusammenhang mit der Sozialisation Jugendlicher fragt Abels (1993) nach dem Erklärungswert einer Normalbiographie angesichts gesellschaftlicher Umbrüche. Er hebt die normierende Bedeutung von Normalitätsmustern hervor, die - trotz aller Individualisierung und Pluralisierung - nach wie vor einen Rahmen bilden, innerhalb dessen sich die individuellen Biographien entwickeln.

In einer vergleichenden Analyse von Untersuchungen der Einstellungen Jugendlicher und junger Erwachsener zur Politik in Ost- und Westdeutschland wird z. B. der Rückgang des politischen Interesses, der in jüngerer Zeit bei dieser Gruppe in Ostdeutschland zu beobachten ist, als „Normalisierung nach der vereinigungsbedingten Mobilisierungsphase“ bewertet (Gille/Krüger/de Rijke/Willems 1996, S. 5, 27). Daneben ist etwa in der Schulforschung von einer Normalisierung der Schulform Gesamtschule die Rede, weil sie sich inzwischen mit den üblichen Problemen öffentlicher allgemeinbildender Schulen auseinander zu setzen hat (Arbeitsgruppe Bildungsbericht 1994, S. 541). Normalisierung wird hier als Beschreibung des 
weitgehenden Erreichens einer akzeptierten Norm benutzt.

Im Zusammenhang mit dem vorletzten Bundesfamilienbericht wird die Forderung nach Normalisierung im Umgang mit Einwanderung erhoben. Die Familienforschung beteilige sich an der Differenzierung zwischen Einheimischen und Zugewanderten, weil Datenbestände nach wie vor fast ausschließlich über deutsche Familien erhoben würden. Normalisierung meint hier eine Änderung der Grenzziehung.

In der Psychologie ist von „Normalisierung“ in manchen Erklärungsmustern die Rede, weil so ebenfalls Ausgrenzungen vorgenommen werden. Eine ähnliche Funktion übernimmt das Normalitätskonstrukt eines Schülers in der allgemeinen öffentlichen Schule. Als Beschreibung der heimlichen Ideal- und Normalvorstellung führt es zum Einbezug derjenigen, die dem Muster entsprechen und zur Ausgrenzung aller, die (zu weit) von dieser Normalität abweichen (Wenning 1996a, 1999).

In der Sozialpädagogik bzw. der Sozialen Arbeit meint Normalisierung etwas ganz anderes, die Frage nach dem (gesellschaftlichen) Stellenwert dieses Arbeitsbereichs (Schaarschuch 1996, S. 861 f.).

Normalisierungen werden in vielen Bereichen gesehen; sie gehören mindestens auf der Beschreibungsebene politischer und wissenschaftlicher Diskussionen - zu den Grundtatbeständen einer modernen Gesellschaft. Eine „Normalität der Normalisierung" liegt in dem Sinne vor, dass entsprechende Prozesse weit verbreitet sind und als normal angesehen werden.

\section{Normalisierung als Prozess der modernen Gesellschaft}

Normalisierung ist in den aufgezeigten heutigen Bedeutungen - historisch gesehen - ein Begriff, dessen Verständnis sich aus wissenschaftlichen, politischen, philosophischen und gesellschaftlichen Veränderungen insbesondere im 18. und 19. Jahrhundert ergibt. Seine inzwischen als schillernd zu bezeichnende Bedeutungsvielfalt erwächst aus unterschiedlichen Verwendungen und Herkünften, die hier kurz skizziert werden.

Normalisierung bezeichnet in wissenschaftlichen Verwendungen in der Regel die "Verrechtlichung" in einem solchen Sinne, wie er etwa in dem Ausdruck, etwas sei „recht und billig“, zu finden ist. Normalisierung bedeutet deshalb, als allgemeinste Beschreibung, die Einführung und Durchsetzung oder die Änderung von Normen bzw. von Normalvorstellungen.

Weil Normalisierung im Rahmen der Entstehung und Verbreitung von Normen verläuft, muss hier auch der Bezug zum Begriff der Norm hergestellt werden. Allerdings wird hier im Wesentlichen als bekannt vorausgesetzt, was in einem weiteren Sinne unter Normen zu verstehen ist, nur einige wichtige Aspekte werden herausgegriffen. In den Sozialwissenschaften existieren unterschiedliche Normbegriffe (Nunner-Winkler 1984, S. 398 ff.): der „statistische“ Normbegriff, der die am häufigsten vorkommende 
Ausprägung - den statistischen Durchschnitt - zur normalen Erwartung bzw. zum verbindlichen Verhalten erklärt, der „soziologische“ Normbegriff der Sozialisationsforschung, der Normen als institutionalisierte Rollenerwartungen begreift, die die Individuen internalisieren, und der „ethische“ Normbegriff, der von einer Subjektvorstellung ausgeht, die das Subjekt als reflexions- und autonomiefähig ansieht.

Für das hier verwendete Verständnis von Norm im Rahmen der Normalisierung reicht die vorwiegend "soziologische“ Idee von Norm aber nicht aus (zum Begriff der „sozialen Norm“ siehe z. B. Schäfers 1992, Peuckert 2000). Gräbt man etymologisch etwas tiefer und führt Normalisierung auf „Normal“ bzw. auf „Normalität“ zurück, zeigt sich in der ursprünglichen Bedeutung ein wichtiger Aspekt, der noch heute mitschwingt: Normen geben Auskunft darüber, wie etwas sein sollte. Deshalb nennt man das, was der Norm nicht entspricht, anormal oder abnorm. Was heute noch bei „abnorm“ mitgedacht wird, geht auf das griechische Verständnis von normal zurück. Im alten Griechenland setzte man normal mit „gesund“ bzw. mit „Natur" gleich. Natur bezeichnete dabei die durchschnittliche, also natürliche Beschaffenheit des Körpers sowie seinen gesunden Zustand. Dieser war zugleich der Idealzustand und das Ziel aller ärztlichen Bemühungen. Das heißt, schon in der ursprünglichen griechischen Bedeutung des Normalen finden sich deskriptive und normative Elemente sowie die Gleichsetzung von normal und natürlich, die bis heute die Mehrdeutigkeit von Norm und Normalität ausmachen (Kudlien/Ritter 1984, Sp. 920).

Wichtiger als die lateinische Bezeichnung norma, die soviel wie Richtmaß, Winkelmaß oder Norm, Regel bedeutet, sind Begriffsveränderungen, die sich u. a. im Umfeld der Französischen Revolution mit ihrer Auflösung „gottgegebener" Hierarchien vollzogen. Die wohl berühmteste Normalitätsvorstellung geht auf die von Gauß 1795 entwickelte Kurve der statistischen Normalverteilung zurück. Sie hat bis heute großen Einfluss auf Normalitätsvorstellungen, etwa im Zusammenhang mit der Verteilung von Intelligenz in der Bevölkerung. Vielleicht nicht zufällig entwickelte Kant zur gleichen Zeit in seiner „Kritik der Urteilskraft“ den Begriff einer „ästhetischen Normalidee“. Er glaubte, durch das Vermessen von vielen Menschen ließe sich ein Durchschnittsbild entwickeln, das ein Schönheitsideal darstellt (Link 1997, S. 195 f.). Ebenfalls 1795 wurden in Frankreich die écoles normales gegründet. Schon 1774 hießen in Österreich die Lehrerausbildungsseminare „Normalschulen“ (ebd., S. 191). Preußen erließ 1763 das Generallandschulreglement, das einen Normal-Lehrplan enthielt, der als Norm galt.

Dennoch: Um 1820 hätte noch niemand sagen oder verstehen können: „Das finde ich ganz normal“ oder „Das ist nicht normal“. 50 Jahre später waren diese Redewendungen dagegen durchaus normal (Link 1997, S. 202).

Für die folgenden Veränderungen spielte Auguste Comte mit seiner 
Idee des Verhältnisses von Krankheit und Gesundheit eine wichtige Rolle. Er entwickelte in der Analyse seines eigenen Wahnsinnsanfalls von 1826 ein Modell des „Normalzustands“. Die von ihm so genannte homöostatische Normalität bezeichnete danach einen normalen psychischen Erregungsgrad, von dem man durch Einflüsse - geistige Überhitzung oder Unterkühlung, wie Comte es beschrieb - abweicht. Bei zu starken Auswirkungen gerät man über die normalen Grenzen hinaus, bis man schließlich, wie er selbst sagte, durchdreht.

Im 17. und 18. Jahrhundert werden vom lateinischen norma (Winkelmaß oder Regel) volkssprachliche Lehnwörter gebildet - im Deutschen wie im Französischen: normal. Das spätere französische normalisation entspricht dagegen dem deutschen Normierung und dem englischen standardization (Link 1997, S. 188). Es geht dabei um mehr als nur eine sprachliche Veränderung: War vorher von Norm und Vernunft die Rede, wenn es um Normalität ging, bezeichnet Normierung und Normalisierung jetzt einen aktiven Prozess der Herstellung von Normalität. Damit wird das Normale dynamisch, veränderbar und es wird als von anderen Faktoren abhängig begriffen, welche mittels Normalisierung eine neue Norm durchsetzen (Kudlien/Ritter 1984, Sp. 925 f.).

An dieser Scharnierstelle der Entwicklung moderner Gesellschaften verändert sich somit auch das Verständnis von Normalität. Sie wird auf diese Weise von etwas Gegebenem zu etwas Beeinflussbarem. Dahinter stehen vermutlich die sich entwickelnde Fortschrittsgläubigkeit, die Entstehung der modernen Wissenschaften und die wachsende Technisierung des Lebens mit der Folge neuartiger „Machbarkeitsvorstellungen“.

Foucaults Studien über Normalität ${ }^{3}$ und Normalisierung setzen mit ihrer Analyse genau an dieser Stelle an. In seinen diesbezüglichen Arbeiten (1976, 1977, auch 1972) betont er den Zusammenhang von gesellschaftlichen Veränderungen mit Normalisierungsprozessen vor allem ab dem 18. Jahrhundert. Er findet, etwa in der École militaire ab der Mitte des 18. Jahrhunderts, anhand von Veränderungen des Strafsystems ein auf verschiedene gesellschaftliche Teilbereiche übertragbares Konzept, das im 18. Jahrhundert entwickelt wurde und das sich im 19. und 20. Jahrhundert verbreitete. ${ }^{4}$ Dieses Konzept besteht aus drei Schritten: Homogenisierung

3 Vor Foucault befassten sich vor allem Georges Canguilhem ab den 1940er Jahren und Theodor W. Adorno mit Normalitätsfragen. Canguilhem und Adorno übertrugen den psychoanalytischen bzw. psychiatrischen Gebrauch von „normal“ auf andere Bereiche. Adorno setzte, vor dem Hintergrund der „Denormalisierung“ zwischen 1914 und 1945, Normalität mit Wahnsinn gleich. Die Mörder in den Konzentrationslagern bezeichnete er, in Anlehnung an die von Hannah Arendt festgestellte „erschreckende Normalität“ Eichmanns, als „Normalungetüme“ (ausführlicher: Link 1997, S. 103 ff.).

4 Wie in „Überwachen und Strafen“ (1976) findet Foucault auch in „Sexualität und Wahrheit“ (1977) im 18. Jahrhundert bzw. um 1800 die entscheidende Schwelle zu Übergängen. Die veränderte gesellschaftliche Entwicklung schlägt sich z. B. in „Bio-Macht“ nie- 
der beteiligten Personen, z. B. durch Gleichstellung (etwa in der Schule), Quantifizierung des Maßstabs, etwa durch Ranglisten (z. B. auf Grund von Punktesystemen zur Bewertung der individuellen Leistung) und Normalisierung mit der Bestimmung des Durchschnitts, eines zu erreichenden Optimums und einer Minimalschwelle, unterhalb derer die Gleichstellung der Beteiligten nicht mehr greift, sondern die Grenze zum Anormalen überschritten ist und eine Ausgrenzung erfolgt (etwa die Definition von geistiger Behinderung auf der Intelligenzskala mit der Folge des Ausschlusses solcher Schüler von „normalen“ Schulen). Neben dem unteren gibt es einen oberen Grenzwert und damit einen normalen Toleranzbereich von Schwankungen. Normalisierungen sind in diesem Zusammenhang dynamisch, sie zielen auf eine Erhöhung des Durchschnitts (schulischer Leistungen, von Produktions- und Arbeitsnormen oder medizinischer Standards usw.) (Link 1997, S. 132 ff.). ${ }^{5}$

Normalität meint im Rahmen dieses Beitrags das Resultat eines Prozesses, der über Alltagsmuster und Routine (Max Weber) bzw. Institutionalisierungen (Arnold Gehlen) hinausgeht. Sie bezeichnet das Ergebnis eines Normalisierungsprozesses, wie er in der modernen Gesellschaft als Reaktion auf deren rasante Dynamik vielfach zu beobachten ist. Zwei kurze Beispiele können dies erläutern: Der Gedanke der Demokratie erfordert etwa die Idee der Gleichheit ${ }^{6}$ und der Unabhängigkeit (vor allem der Wähler in einer demokratischen Institution sowie der verschiedenen gesellschaftlichen Institutionen, auch eine Gleichheit der Staaten). Dabei muss immer die Frage von Zugehörigkeit und Nichtzugehörigkeit - also der Grenzziehung - geklärt werden: Wer hat wann das Recht zu wählen? bzw. Wer gehört zu dem Staat und wer nicht? Auf einer anderen Ebene gibt es bei-

der, die die Körper in Produktionsapparate einpassen und generell stärker nutzen will, etwa durch die Unterstützung des Anwachsens der Bevölkerungszahl (siehe auch den Beitrag von Maurer in diesem Band).

Auch Marcuses Analyse des „eindimensionalen Menschen“ (1967) kann normalisierungstheoretisch so gelesen werden, dass sie eine der ersten grundlegenden Auseinandersetzungen mit Normalisierungsmechanismen ist. Der Begriff „Eindimensionalität“ bezieht sich z. B. auf die wissenschaftliche Konstruktion von Homogenitäten, die durch das Ausklammern „unpassender“ Daten und Faktoren „hergestellt“ werden. Auch andere Normalisierungsmuster in Form der Verbreitung geänderter „Normalitäts-Entwürfe“, z. B. durch Film und Fernsehen, geraten schon in Marcuses Blickfeld. Selbst der Begriff „Normalisierung" ist enthalten (S. 97), wenn auch noch in einer psychischen Dimension. Damit stand Marcuse vor einer „Theorie des flexiblen Normalismus" (Link 1997, S. 118), wie sie in der These der repressiven Toleranz als neuem Reaktionsmuster der Integration von (gesellschaftlichen) Abweichungen durch Vereinnahmung anklingt. Marcuse hat sich mit sektoriellen Normalitäten befasst, von denen es nur noch ein Schritt gewesen wäre, ,[...] Normalität und Normalisierung als interdiskursive, kulturell hegemoniale Strategie systematisch [...] in den Blick fassen [zu] können“ (ebd.).

$\mathrm{Zu}$ beachten ist, dass Demokratie nie die Gleichheit aller meint bzw. bewirkt, immer wird Inklusion und Exklusion betrieben. So wurde z. B. in fast allen Staaten das politische Wahlrecht für Frauen erst lange nach dem Wahlrecht für Männer eingeführt. 
spielweise die (industrie-)gesellschaftliche Forderung nach einer funktionierenden unpersönlichen Kommunikation, d. h. nach gelingenden Mitteilungen von Menschen, die sich weder kennen noch direkt miteinander reden (z. B. für ein Telefongespräch mit Unbekannten oder für eine schriftliche Bedienungsanleitung). Dies erfordert eine „Normalisierung“ der Sprache; Kommunikation muss auch ausschließlich mit Hilfe der Aussagekraft der Zeichen und Worte sinnvoll möglich sein (Kremnitz 1997). Die durch diese Ansprüche entstandene „Normalität" ist in dem einen Fall in Deutschland (vereinfacht gesagt) die Beschränkung des Wahlrechts auf alle volljährigen Personen mit deutscher Staatsangehörigkeit; in dem anderen Fall ist es die (unhinterfragte, weil als normal betrachtete) Annahme einer von allen beherrschten Standardsprache in normalen Kommunikationssituationen, etwa im Unterricht der öffentlichen Schule oder in Behördenkontakten, der so genannte „monolinguale Habitus“ (Gogolin 1994).

Normalisierung bezeichnet im Rahmen dieser Fragestellung deshalb den Prozess der Herstellung einer Normalität. Er ist der individuelle bzw. gesellschaftsweite Prozess der Anpassung mentaler und/oder realer Muster an neue/veränderte Normalitätsvorstellungen sowie die Herausbildung oder Ausdifferenzierung solcher Vorstellungen Da dieser Prozess immer unter bestimmten historischen und sozialen Bedingungen abläuft, ist jede Normalisierung die Durchsetzung einer neuen Normalität, die eine andere verdrängen muss, um „normal“ sein zu können. Normalisierung und in der Regel zu Beginn auch die neue Normalität haben deshalb wenig mit Durchschnitt oder Alltäglichkeit zu tun, aber viel mit Machtinteressen und Sanktionsmöglichkeiten. In der modernen Gesellschaft mit ihren (scheinbar) individualisierten Chancenzuschreibungen sind dazu weniger Gesetze und Polizeigewalt notwendig als vielmehr Diskurskontrolle der Massenmedien und Verknappung von Chancen (etwa auf entlohnte Arbeit). Die Gefahr der individualisierten Schuldzuweisung für mangelnden Erfolg bei der gesellschaftsbezogenen Realisierung von Chancen bietet mehr und nachhaltigere Möglichkeiten der Beeinflussung durch die Mächtigeren.

Als Normalisierungsmuster werden hier dementsprechend wiederkehrende oder verbreitete Normalisierungsprozesse bezeichnet. Bestimmte Normalitätsvorstellungen und ihre Herstellung scheinen systematisch aufzutreten und verdichten sich zu solchen Normalitätsmustern. Normalisierungsmuster sind Prozesse der gesellschaftlich relevanten Durchsetzung impliziter und expliziter sozial wirksamer Maßstabsdefinitionen.

Die Notwendigkeit gesellschaftlicher Relevanz der Normalisierungsmuster ergibt sich aus dem sozialen Charakter von Normen; dass Normalisierung die aktive Durchsetzung von Standards ist, die eben auch anders sein könnten, d. h. dass sie eine Frage der Machtverteilung ist, sollte ebenfalls klar geworden sein. Die Kennzeichnung als Durchsetzung von Maßstabsdefinitionen zielt darauf, dass die Durchsetzung eines Maßstabs oder eines 
neuen Maßstabs die Verdrängung eines anderen oder eines alten zur Folge hat, was selten konfliktfrei abläuft. Außerdem liegt die gesellschaftliche Relevanz in der Anwendung des Maßstabs, d. h. in seiner sozialen Wirksamkeit. Die Durchsetzung des neuen Maßstabs bewirkt eine Ausgrenzung derjenigen, die ihm nicht entsprechen, einerlei ob sie es nicht können oder nicht wollen. Dabei spielt nur eine kleine Rolle, ob der Maßstab ausdrücklich und offen vertreten wird oder ob er im Verborgenen wirkt.

Normalisierungsmuster lassen sich mit Grenzziehungen vergleichen: räumliche und soziale Grenzen schließen zugleich ein und aus. Die Gren zen des Nationalstaates und das Instrument der Staatsbürgerschaft produzieren beispielsweise erst Inländer und Ausländer. Die sozial wirksamen Grenzziehungen, d. h. die Mechanismen der Inklusion und Exklusion, ${ }^{7}$ sind von der spezifischen sozialen Ordnung abhängig, in der sie vollzogen werden - für das deutsche Bildungswesen ist z. B. die Abhängigkeit von formalen Qualifikationen, das sogenannte Berechtigungswesen, wichtig.

\section{3 „Normale" pädagogische Theorie}

In der Erziehungswirklichkeit ließe sich auf Anhieb eine Fülle von Normalisierungen entdecken. Alle Übereinkünfte, die auch anders geregelt sein könnten, stehen unter dem Einfluss von Normalitätsvorstellungen. In der Wissenschaft von der Erziehung sind ebenfalls seit langer Zeit implizite Normalitätsvorstellungen und Normalisierungen vorhanden. Diese impliziten Normalisierungen werden auf eine verdeckte Weise bis heute fortgesetzt. Deren unbedachte „Vererbung“ ist ein Normalisierungsmuster, auf das hier hingewiesen wird.

Als Beispiel, das in der pädagogischen Lehre immer noch eine große Bedeutung besitzt, dient dafür der Erziehungsroman Émile von Jean-Jacques Rousseau. Zur Einschätzung dieser Schrift der Klappentext:

„Der »Emil« ist ein Epochenwerk, das wegen seiner fundamentalen Bedeutung für unser pädagogisches Menschenbild zum Pflichtpensum jedes Lehrers und Erziehers gehört. Das um so mehr, als es - heute wie bei seinem ersten Erscheinen im Jahre 1762 - eine erfrischend provozierende Lektüre [...] bleiben wird. Die pädagogischen Reformen der letzten 200 Jahre sind nicht denkbar ohne Rousseaus Entdeckung des Kindes. Dieser Herausforderung wird sich auch der moderne Leser nicht entziehen können“ (Rousseau 1762, Klappentext).

Rousseaus Art der Entdeckung des Kindes ist aus heutiger Sicht, wie ich zeigen möchte, eher provozierend als erfrischend. Rousseau wählt sich seinen Zögling als einen „Durchschnittsgeist“ (Rousseau 1762, S. 26), weil nur der Durchschnitt Erziehung brauche und als Beispiel dienen könne. Die

Siehe zur Bedeutung dieser Mechanismen im soziologischen Erklärungsansatz der Systemtheorie z. B. den Beitrag von Opp, Fingerle und Puhr in diesem Band. 
anderen erziehen sich, nach seiner Ansicht, selbst. Die zweite Begründung für die getroffene Wahl des Zöglings: Da die „Durchbildung des Gehirns“ (ebd., S. 27) im Norden wie im Süden weniger vollkommen sei als im zentralen Europa, wählt sich Rousseau seinen „Weltbürger [...] aus der gemäBigten Zone; aus Frankreich zum Beispiel eher als anderswoher" (ebd.). Die dritte Begründung: Rousseau geht zwar davon aus, dass die propagierte natürliche Erziehung für alle Lebensumstände tauglich mache, er hält aber nur die Erziehung der Reichen für erforderlich, da der Arme durch seinen Stand erzogen werde und andere Bemühungen sowieso unnütz, weil unvernünftig seien. Er hätte auch nichts dagegen, wenn sein Zögling adlig wäre. Es zeigt sich: Der gewählte Zögling ist gerade nicht durchschnittlich, weder zu Rousseaus Zeit noch heute.

Weitere Prämissen zeigen sich z. B. in einer Nebenbemerkung: „Emil wird ein ebenso reines Französisch sprechen, wie ich es vermag ..." (Rousseau 1762, S. 51). Die Bevorzugung eines rein französischsprachigen Jungen ist für den Genfer Uhrmachersohn, der auf seinem Lebensweg bis zur Abfassung seines Erziehungsromans auch in anderssprachigen Regionen gelebt hat, nicht ohne Weiteres zu erklären. Im damaligen Frankreich sprach darüber hinaus die Mehrheit der Franzosen kein Französisch und die übrigen ein anderes als das Französisch der Pariser, der kulturellen und Machtmetropole. Rousseau geht deshalb auch von einer sprachlichen Wunschvorstellung aus, wenn er seinen „Durchschnittsgeist“ beschreibt.

Eine weitere Prämisse zeigt die geschlechtsspezifische Differenzierung der Erziehung. Zwar geht Rousseau von einer natürlichen Ordnung aus, in der alle Menschen gleich sind und in der für alle die gemeinsame Berufung sei, Mensch zu sein (Rousseau 1762, S. 14), Unterschiede zwischen Männern und Frauen sieht er aber schon in deren Bestimmung:

„Steht dieser Grundsatz fest, so folgt daraus, daß die Frau eigens geschaffen ist, um dem Mann zu gefallen“" (Rousseau 1762, S. 386).

„Nachdem einmal bewiesen ist, daß der Mann und die Frau weder nach dem Charakter noch nach dem Temperament gleich gebildet sind noch sein dürfen, so folgt daraus, daß sie auch nicht die gleiche Erziehung haben dürfen“ (ebd., S. 392).

Die skizzierten Normalitätsvorstellungen ${ }^{8}$ sind zudem um einen Aspekt zu erweitern, der oft übersehen wird (Bundschuh 1997, S. 310):

„Ich würde mich nicht mit einem kränklichen und siechen Kind belasten, und wenn es achtzig Jahre alt würde. Ich mag keinen Zögling, der sich selbst und anderen unnütz ist, der allein damit beschäftigt ist, sich am Leben zu erhalten, und dessen Leib der Erziehung der Seele schadet. Verschwende ich meine Fürsorge an ihn, so verdopple ich den Verlust, indem ich der Gesellschaft zwei statt nur einen Menschen entziehe“ (Rousseau 1762, S. 32).

Verschiedene Beiträge in Rendtdorff/Moser (1999) greifen die Konstitution der Zweigeschlechtlichkeit bei Rousseau auf. 
Aus scheinbaren Nützlichkeitserwägungen lehnt Rousseau die Erziehung behinderter und gebrechlicher Kinder ab. Er glaubt nicht an ihre Fähigkeit zu geistiger Entwicklung. Dies könnte man den Utilitätsbestrebungen zuschreiben, zumal Rousseau ja nur für seine eigene Person $\mathrm{zu}$ sprechen scheint. Der Émile ist demgegenüber aber, wie die aufgezeigten Charakteristika belegen, tief von Normalitätsvorstellungen geprägt, die - wenn die Schrift auch als Utopie angelegt war - wenig mit der damaligen Realität zu tun haben. Unser heutiges Normalitätskonstrukt über den normalen Schüler beruht vermutlich zu einem erheblichen Teil auf derart weiter getragenen Normalitätsvorstellungen. Dieses hier nur angedeutete heimliche Ergebnis der Tradierung pädagogischer Werke ist ein wichtiger Aspekt für die Wirkungen von Normalisierungsmustern im Bildungswesen.

Um dies klarzustellen: Hier soll weder Rousseau denunziert noch ein über 200 Jahre alter Text umstandslos dem Licht heutiger Aufmerksamkeiten ausgesetzt werden. Es geht vielmehr darum, dass es sich um einen nach wie vor zur Lektüre empfohlenen Pädagogen bzw. um ein verbreitetes pädagogisches Werk handelt. Problematisch ist, vor dem Hintergrund des gezeichneten Bildes eines Zöglings, diesen Erziehungsroman heute in einem positiven Sinne als „Epochenwerk [...] mit fundamentaler Bedeutung für unser pädagogisches Menschenbild“ (Rousseau 1762, Klappentext) zu bezeichnen und als vorbildlich zu charakterisieren. Darin enthaltene pädagogische Theorien wirken, unkritisch verbreitet, normalisierend im erläuterten Sinne. Sie können auf diese Weise ein pädagogisches Menschenbild prägen und (unbedacht?) als Normalisierungsinstrument benutzt werden.

\section{$4 \quad$ Subdisziplinbildung und Anspruch auf Allgemeingültigkeit}

Normalisierungsmuster sind in pädagogischen Theorien implizit und explizit enthalten. Daneben sind solche Muster auch an ganz anderen Stellen $\mathrm{zu}$ finden. So kann man etwa die gegenwärtige Struktur der (bundesdeutschen) Erziehungswissenschaft, insbesondere bestimmte neuere subdisziplinäre Entwicklungen, entsprechend interpretieren. Die Erziehungswissenschaft bzw. Pädagogik differenzierte sich in Deutschland in den letzten Jahrzehnten breit aus. Die Aufgliederung und Aufspaltung geht inzwischen so weit, dass von Unübersichtlichkeit und nicht mehr herzustellendem $\mathrm{Zu}$ sammenhang der Einzelteile die Rede ist (siehe Lenzen 1989, S. 1114 f., Lenzen 1999). Gefragt wird in diesem Rahmen, ob bestimmte Formen der Subdisziplinbildung der Erziehungswissenschaft auf Normalitätsvorstellungen zurückzuführen sind und als Wirkung eines impliziten Normalitätskonstrukts über den Gegenstand der Wissenschaft begriffen werden können. Wäre dies so, dürften allgemeine erziehungswissenschaftliche Theorien keinen Anspruch auf Allgemeingültigkeit mehr in dem Sinne erheben, dass ihr Gegenstandsbereich die Erziehungs- und Bildungstatsache 
aller zu Erziehenden und Bildenden ist.

Interkulturelle Erziehungswissenschaft, Feministische Pädagogik und Integrative Pädagogik gelten als Problemreaktionen der Erziehungswissenschaft bzw. der Pädagogik. Wenn man diese Sichtweise akzeptiert, stellt sich die Frage: Was ist das Problem, auf das diese subdisziplinären Entwicklungen eine Antwort darstellen?

Weil in diesem Band die Feministische Pädagogik im Beitrag von Löw und die Sonderpädagogik in dem von Opp, Fingerle und Puhr angesprochen werden, ${ }^{9}$ geht es nachfolgend um das Beispiel der Interkulturellen Erziehungswissenschaft. Innerhalb dieser Subdisziplin besteht ein breiter Konsens über die Ursache, auf die deren Entwicklung zurückzuführen ist.

„Generell wird interkulturelle Erziehung gegenwärtig verstanden als pädagogische Reaktion, theoretischer und praktischer Art, auf die migrationsbedingte kulturelle Pluralität der Gesellschaft" (Hohmann 1989, S. 12).

Die Gegenstandskonstitution dieser subdisziplinären Neubildung wird also auf Migration zurückgeführt. Damit ist als Folge die Annahme einer kulturellen Pluralisierung der Gesellschaft verbunden. Durch die Zuwanderung über Staatsgrenzen hinweg verändert sich danach die kulturelle Zusammensetzung der aufnehmenden Gesellschaft. Soweit ist der Gedanke nachzuvollziehen. Er beruht aber auf fragwürdigen Annahmen: die Aufnahmegesellschaft sei zuvor kulturell homogen gewesen und Migration sei ein neues Phänomen. Diese Annahmen sind nicht stichhaltig: kulturelle Pluralität existierte in der Gesellschaft schon vor und auch neben der Pluralisierung durch Zuwanderung; Migration ist zudem ein altes und verbreitetes soziales Phänomen (siehe dazu z. B. Wenning 1996b).

Der Grund für die Entwicklung einer Interkulturellen Erziehungswissenschaft muss darum woanders gesucht werden. Man kann diese Subdisziplin auch als Reaktion auf das zunehmende Versagen der tradierten gesellschaftlichen Homogenisierungsmuster begreifen (Krüger-Potratz 1994). Der bis dahin praktizierte Umgang mit kultureller Pluralität war nicht mehr aufrecht zu erhalten, ohne die Legitimität des Systems zu gefährden. Die gesellschaftliche „Homogenisierungsmaschine“ Schule geriet ins Stottern und verlangte nach Hilfe. Die Reaktion der Erziehungswissenschaft auf diese Entwicklung erfüllt die Merkmale des oben beschriebenen Normalisierungsprozesses. Am Anfang steht die Homogenisierung durch die, zum Teil von außen erzwungene, Gleichstellung aller Kinder und Jugendlichen gegenüber der Institution Schule durch die Schulpflicht für alle (Beschluss der Kultusministerkonferenz zum Unterricht für Kinder von Aus-

Zum Verhältnis der Kategorien Geschlecht und Behinderung zu Normalität siehe auch Schildmann (2000). Unter Rückgriff auf die Argumentation Links (1997) findet sie Parallelen zwischen weiblichem Geschlecht und Behinderung als sozialen Kategorien in der Abweichung von gesellschaftlichen Normalitätsvorstellungen. 
ländern 1964). Quantifizierung und Normalisierung erfolgten durch das Schulwesen mit dem Ergebnis des mangelnden Erfolgs bestimmter Gruppen von Schülern und Schülerinnen. Diese Gruppen konstituierten sich nicht entlang der anerkannten Grenzen von Begabung oder schulischer Leistungsfähigkeit, sondern wurden durch schulisch irrelevante Faktoren, insbesondere die abweichende Staatsangehörigkeit, identifiziert. Dadurch geriet das schlechte Abschneiden der Kinder aus diesen Gruppen zum bildungspolitischen Skandal. Gleichzeitig bekam die erziehungswissenschaftliche Theoriebildung mit ihrem Anspruch auf Allgemeingültigkeit Erklärungsschwierigkeiten, weil sie den Problemen der als neu wahrgenommenen Gruppen hilflos gegenüber stand, bzw. diese nicht in den Blick nahm.

So verstanden kann man die darauf folgende Etablierung einer Interkulturellen Erziehungswissenschaft als Ausgrenzungsprozess begreifen: Die herrschende Normalitätsvorstellung wird angesichts der Infragestellung gerettet, indem das zu sehr Abweichende als etwas Eigenes definiert wird, das jenseits der Grenze des „Normalen“ liegt. Dafür ist dann eine gesonderte wissenschaftliche Spezialisierung zuständig. ${ }^{10}$ Die Nebenfolge dieser Entwicklung: Die bestehenden erziehungswissenschaftlichen Disziplinen existieren unhinterfragt und „ungestört" weiter. Zudem werden sie in ihren grundlegenden Vorstellungen - d. h. in ihren Normalitätsannahmen - bestätigt, weil es für die zu sehr abweichenden Erscheinungsformen eigene Zuständigkeiten gibt. Die neue Subdisziplin entlastet auf diese Weise die übrige erziehungswissenschaftliche Theoriebildung.

Mit der jüngeren Subdisziplinbildung der Interkulturellen Erziehungswissenschaft untergräbt die allgemeine erziehungswissenschaftliche Theorieentwicklung - so eine hier zugrunde liegende These - indirekt ihren Anspruch auf Gültigkeit als „Normalpädagogik“. Denkt man diesen Prozess weiter und bezieht die anderen subdisziplinären Entwicklungen Feministische Pädagogik und Sonderpädagogik ein, erhebt sich die Frage: Wie allgemein ist die allgemeine pädagogische Theoriebildung? Ist der Anspruch auf Allgemeingültigkeit nur noch der Anspruch, den eine Art „Sonderpädagogik für Normale" erheben kann? Sie ist, vor einem solchen Hintergrund, als eine Pädagogik für die „Normalen“ nach dem jeweils gültigen Normalitätskonstrukt (Wenning 1999, S. 328 ff.) zu beschreiben. Damit sind allgemeine pädagogische Theorieentwürfe variabel und nur noch zeitgebundener Ausdruck der jeweils aktuellen partikularen Interessen der „Normalen“, d. h. derjenigen, die zur fraglichen Zeit über die Macht verfügen, ihre

Hierbei ist wichtig, dass die erste Reaktion der Erziehungswissenschaft auf diese Problemwahrnehmung als eine „Sonderpädagogik für Kinder mit anderer Staatsangehörigkeit“, als Ausländerpädagogik, zu kennzeichnen ist. Die heute verfolgten Ziele einer modernen Interkulturellen Erziehung beziehen sich auf die für alle veränderte Situation der größeren sprachlichen, kulturellen, religiösen ... Pluralität und den Umgang damit. Eine derartige Spezialisierung ist eine Ausdifferenzierung einer Allgemeinen Erziehungswissenschaft, bzw. deren Ergänzung, und keine „Sonderpädagogik“. 
Maßstabsdefinitionen durchzusetzen (Wenning 1993, S. 238 f.). So erklärt sich z. B. das verengte Bild, das Rousseau von seinem Musterzögling zeichnete. Aus dem gleichen Grund sind die klassischen pädagogischen Theorien, die im aufkommenden bürgerlichen Zeitalter entstanden, bürgerliche Pädagogiken und keine wirklich allgemeinen Theorien. Indem sie ihren „Kanon“ fortschreiben, legen sie fest, was zu lernen und damit wichtig ist.

Die jüngere erziehungswissenschaftliche Subdisziplinbildung kann als Reaktion auf das Infragestellen erziehungswissenschaftlicher Normalitätsvorstellungen zurückgeführt werden (siehe auch die Einleitung zu diesem Buch). Die Herausbildung dieser Disziplinen stützt vorläufig die verbreiteten Ideen über das Normale - im Widerspruch zur empirisch fassbaren Realität. Deshalb stellt diese Subdisziplinbildung im gleichen Maße ein Normalisierungsmuster dar.

\section{$5 \quad$ Interkulturelle Erziehungswissenschaft: Problemursache oder Problemlöserin?}

Die Frage nach Berechtigung und Wirkungen der subdisziplinären Aufgliederung einer Wissenschaft ist auf den ersten Blick eine rein akademische Angelegenheit, eine Beschäftigung der Wissenschaft mit sich selbst - interessant höchstens für Wissenschaftstheoretiker und andere Bewohner des Elfenbeinturms. Vor dem Hintergrund der Frage nach Normalisierungsmustern stellt sich dies anders dar. Auf zwei Ebenen, auf der Disziplinebene und auf einer „Wirkungs-“ sprich Praxisebene, lässt sich verdeutlichen, dass solche Ausdifferenzierungen weitreichende Folgen haben können. Als Beispiel geht es wieder um die Interkulturelle Erziehungswissenschaft.

Ein wissenschaftliches Interpretationsangebot in Form einer Subdisziplin mit eigenen Theorieangeboten wirkt unter Umständen weniger aufklärerisch als, möglicherweise, „verklärerisch“. Ein solches Angebot kann, über die im letzten Abschnitt angesprochenen Wirkungen auf der Ebene der Theorieentwicklung hinaus, normalisierende Ergebnisse hervorrufen, indem es Erklärungsmuster dominiert und problematische soziale Folgen unterstützt oder sogar hervorruft. Die Existenz bestimmter Theorien hat Auswirkungen auf die Untersuchungsergebnisse über soziale Realitäten. Der wissenschaftliche Blick wird durch die angewandten Methoden und den wissenschaftstheoretischen Hintergrund geprägt. Dazu zählen die existierenden und als „gültig" anerkannten Theorien. Je nach „Lesart" (Koller 1999) eines sozialen Phänomens ergibt sich ein anderes wissenschaftliches Bild. Mit Lesarten sind die verschiedenen möglichen wissenschaftlichen Zugänge gemeint. Dabei gibt es nicht das eine, richtige Bild, welches die Wahrheit wiedergibt.

Die Gefahr besteht, dass, im Gegensatz zu dieser Erkenntnis, das Er- 
gebnis der einen vorliegenden plausiblen Untersuchung als die einzig wahre Erklärung angenommen wird. So entstehen wissenschaftliche Weltbilder über bestimmte soziale Phänomene mit dem ihnen eigenen Beharrungsvermögen. Beispiel: Wenn man sich einen Untersuchungsgegenstand mit den Schlüsselbegriffen „schulische Erziehung“, „Ausländer“ und vielleicht noch „verschiedene kulturelle Muster" vor Augen führt, denken - in erziehungswissenschaftlichen Zusammenhängen - viele vermutlich sofort an Interkulturelle Erziehungswissenschaft (bzw. Ausländerpädagogik). ${ }^{11}$ Die Existenz dieser Spezialisierung lenkt die Aufmerksamkeit und beeinflusst die Wahrnehmung. So bekommen die theoretischen Erklärungsansätze dieser Spezialisierung eine besondere Bedeutung.

Stellt man sich weiter vor, eine Interkulturelle Erziehungswissenschaft existierte nicht, so würden andere theoretische Ansätze für das fragliche soziale Phänomen assoziiert. Man könnte sich z. B. mit dem Schulerfolg der Kinder befassen und zu dem Schluss kommen, die lange bekannte Abhängigkeit des Schulerfolgs von der Sozialschicht bzw. der Bildung der Eltern gälte noch immer oder nehme wieder zu. ${ }^{12}$ Die Staatsangehörigkeiten des Untersuchungsklientels müssen dabei nicht interessieren, weil diese für schulische Lernprozesse an sich bedeutungslos sind.

Es lohnt sich also, die Deutungsmuster der Interkulturellen Erziehungswissenschaft von einer Metaebene aus zu betrachten. Beispiel: Seit etwa einem Jahrzehnt gibt es in der sozialwissenschaftlichen Diskussion eine Argumentationslinie (z. B. Bukow/Llaryora 1988/1993, Lutz 1999a), die hervorhebt, der wissenschaftliche Diskurs sei an der Konstruktion von Eingewanderten als „ethnischen“ Minderheiten beteiligt. Dieser Ethnisierungsprozess ${ }^{13}$ läuft danach so $\mathrm{ab}$, dass bestimmte Deutungsmuster verallgemeinert werden. Dies hat zur Folge, dass zunächst belanglose Elemente „schrittweise in konstitutiv relevante Eigenschaften transformiert werden, um eine gesonderte soziale Gruppe zu erzeugen“ (Bukow 1996, S. 63). Der

11 Zum Verhältnis von Interkultureller Erziehungswissenschaft und Ausländerpädagogik siehe Lutz (1999b).

Siehe dazu kurz zusammengefasst die entsprechende Auswertung der 15. Sozialerhebung des Deutschen Studentenwerk von Müssig-Trapp (2000).

13 Allerdings ist dabei schon der Begriff Ethnizität problematisch. So sieht etwa Lentz (1998) bei ihrer Analyse der Konstruktion von Ethnizität in Ghana diese als eine Kategorie an, die sich nicht eindeutig definieren lässt: „Ethnizität ist vielmehr ein schillerndes Konzept, das längst nicht mehr nur zum Repertoire von Sozialwissenschaftlern, sondern auch zum handlungsrelevanten Wortschatz von Häuptlingen, Politikern, lokalen Intellektuellen, Arbeitsmigranten und sozialen Bewegungen gehört. Der Leser wird, so hoffe ich jedenfalls, am Ende des Buchs wissen, welche Akteure was (und warum) unter ethnischer Identität verstanden und wie sie dieses Verständnis praktisch umgesetzt haben. Als Arbeitsdefinition soll hier der Hinweis genügen, daß Ethnizitätsdiskurse generell essentialistisch argumentieren und gesellschaftliche Verhältnisse naturalisieren, indem sie Abstammung und gemeinsame Herkunft, in Kombination mit Sprache, Kultur und Territorium, zum entscheidenden Konstituens von Gemeinschaft erklären“ (Lentz 1998, S. 31). 
Indikator Staatsangehörigkeit bietet sich besonders an, um eine dominante Gruppe - die Einheimischen - von einer machtdefizitären Gruppe - den Zugewanderten - zu unterscheiden. Den so entstandenen Gruppen werden Attribute zugeordnet, die dann normalisiert werden, d. h. die die Wahrnehmung und die Bewertung der Gruppen bestimmen und Abweichungen von diesen Bildern ausblenden. Als letzter Schritt erfolgt die eigentliche Ethnisierung, indem die Resultate des bisherigen Prozesses zu wesenhaften und somit unentrinnbaren Zügen der Gruppen werden. Den Mitgliedern der so konstituierten Gruppen - seien es nun „die“ Deutschen, „die“ Türken oder „die“ Asylbewerber - werden ethnische Eigenschaften, aber auch differente rechtliche sowie kulturelle Ausstattungen zugewiesen. Der Ethnisierungsprozess gewinnt soziale Relevanz, indem die Beteiligten sich in ihrem Selbstverständnis, der gegenseitigen Wahrnehmung und entsprechendem Handeln an der Existenz solcher Gruppen orientieren und sie damit zu sozialer Realität werden lassen. Das gilt für die Mitglieder der dominanten Gruppe wie für die Mitglieder der dominierten Gruppen (ebd., S. 64 ff.).

Hier setzt die Kritik eines Teils der interkulturellen Erziehungswissenschaft an (insbesondere Bommes/Radtke 1993, Lentz 1994, Radtke 1995, Diehm/Radtke 1999). Konkret lautet der Vorwurf: Auf der Ebene der Disziplin trage die Subdisziplinbildung zu einer wissenschaftlich gestützten Ausgrenzung bei. ${ }^{14}$ Das Aufgreifen von ethnischen Differenzen führe selbst zur Ethnisierung:

„Die im Programm der ,interkulturellen Erziehung' zugrunde gelegten, wesentlich ethnisch verstandenen Differenzen zwischen Schülern sind nicht schlicht gegeben, sondern werden in ihrer sozialen Bedeutsamkeit erst hergestellt" (Bommes/Radtke 1993, S. 487).

Mit den Aktivitäten der interkulturellen Erziehung würden zudem gesellschaftliche Ersatzhandlungen inszeniert. Im Ergebnis lenke dies von den eigentlichen gesellschaftlichen Problemen ab (Radtke 1995, S. 856).

Ähnliches trifft die antirassistische Erziehung, wie sie insbesondere in

14 Auch in der Kulturanthropologie gibt es seit einiger Zeit Diskussionen darüber, dass die Hervorhebung bzw. Untersuchung bestimmter, als „anders“ angesehener Kulturen zu einem othering der damit gekennzeichneten Personen oder Gruppen führt, dass so Differenz größer gemacht, d. h. konstruiert, wird (siehe z. B. Schiffauer 1997, S. 164).

Waldenfels $(1998$, S. 9) beschreibt demgegenüber mit der Normalisierung des Fremden eine Strategie im Umgang mit dieser Wahrnehmung: Indem man das Fremde in den normalen Gang der Dinge einordnet, verliert es den Status des Außergewöhnlichen. Mit dieser Strategie lässt es sich „bewältigen“ und man kann sich selbst beruhigen.

Die scheinbar gegensätzlichen Aussagen, hier die Vergrößerung von (kultureller) Differenz, da die Normalisierung (kultureller) Differenz, sind vereinbar, weil sie, wenn zum Teil auch gegenläufig, auf unterschiedlichen gesellschaftlichen Ebenen angesiedelt sind. Betrachtet man die Reaktion auf die Wahrnehmung von Fremdheit als Normalisierungsprozess, sind sie zudem verschiedenen Phasen des oben erläuterten Normalisierungsvorgangs zuzuordnen. 
Großbritannien und den Niederlanden als Gegenpol zur interkulturellen Erziehung entwickelt wurde. Sie soll einen Rassialisierungsprozess in Gang gesetzt haben: die Unterscheidung zwischen „Einheimischen“ und „Zuge-

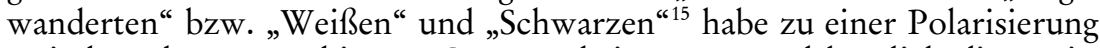
zwischen den so markierten Gruppen beigetragen und letztlich die rassistisch begründete Gewaltbereitschaft auf beiden Seiten erhöht (SteinerKhamsi 1996, S. 269 f.)

Antirassistische wie interkulturelle Erziehung benutzen (vermeintliche) Gruppenmerkmale für ihre Argumentation, die sie eigentlich abschaffen oder unwirksam machen wollen. Sie sind essentialistisch, weil sie bestehende oder vermeintliche Unterschiede $\mathrm{zu}$ Wesenszügen von sozialen Gruppen erheben und sie quasi als natürlich gegeben voraussetzen. Darauf bauen sie ihre Daseinsberechtigung auf. Die Folgen gelten auch für jede Praxis, die Gruppenunterschiede polarisiert (Steiner-Khamsi 1996, S. 270 f.). Die Hervorhebung kultureller oder ethnischer Unterschiede durch wissenschaftliche Erklärungsansätze hat eine normalisierende Wirkung, die tief in die Bildungspraxis hineinreicht. Dies zeigt sich etwa an der Lehrerin, die ihren Konflikt mit einem pubertierenden Nachwuchsmacho fast automatisch als Kulturkonflikt interpretiert, wenn es sich z. B. um einen türkischen Jungen handelt.

Damit stellt sich die Frage, ob Interkulturelle Erziehungswissenschaft die Problemlöserin oder die Problemursache ist. Schafft sie durch ihre Existenz die Schwierigkeiten erst, die sie dann vorgibt, lösen zu wollen? Oder waren die Probleme schon da und diese wissenschaftliche Auseinandersetzung hat sie nur aufgegriffen? Die Konsequenz wäre in dem einen Fall die Abschaffung der Interkulturellen Erziehungswissenschaft und das Ende interkultureller Erziehungsbemühungen. Im anderen Fall sollte die Interkulturelle Erziehungswissenschaft ausgebaut werden, weil die Problemlagen, auf die sie reagiert, zunehmen und sich verschärfen.

Der Widerspruch kann und soll an dieser Stelle nicht ausdiskutiert werden. Die „Wahrheit“ liegt - wie so oft - vermutlich in der Mitte. Allein die Möglichkeit der Produktion von Differenz durch diese wissenschaftliche Spezialisierung sollte Anlass zu Vorsicht sein. Wissenschaftliche Interpretationsangebote haben eine normalisierende Wirkung auf die Wahrnehmung und die Interpretation sozialer Realität. Die Wirkung kann bis in alltagstheoretische Erklärungen hinein reichen und soziales Handeln bestimmen.

Die Ethnisierung bzw. Kulturalisierung von Konflikten kann dazu beitragen, diese auf eine andere Ebene zu verschieben und von den eigentlichen Ursachen abzulenken. Das Hervorheben von Gemeinsamkeiten ist genauso möglich wie die Betonung von Unterschieden; es hängt von den verfolgten Absichten ab (Hansen 1996, S. 112 f.). Eine Alternative zum

Siehe auch den Beitrag von Leiprecht und Lang in diesem Band zu entsprechenden unbedachten Folgen eines gut gemeinten Antirassismustrainings. 
Schaffen oder Betonen von Unterschieden wäre ein allgemeiner Perspektivwechsel hin zur Akzeptanz von Vielfalt als Normalzustand und der Abkehr von Einheitlichkeitsvorstellungen (Hansen 1994, S. 194, siehe dazu auch den Beitrag von Prengel in diesem Band).

Wissenschaftliche Theorien können - das sollte hier deutlich werden normalisierende Wirkungen haben. Dies zeigt sich in der Vorstrukturierung der Analyse von Untersuchungsgegenständen und indirekt in der alltagstheoretischen Wahrnehmung sowie in entsprechendem Handeln. Dies gilt nicht nur für die als Beispiel herangezogene Interkulturelle Erziehungswissenschaft. Möglicherweise kann man alle wissenschaftlichen Paradigmenwechsel als neue Normalisierungen begreifen - und damit ihnen gegenüber kritisch bleiben.

Insgesamt zeigt dieser Beitrag auf, dass das Argumentationsmuster „Normalisierung" weit verbreitet ist. Daneben wird deutlich, dass Normalisierungsmuster als Durchsetzung gesellschaftlich relevanter impliziter wie expliziter Maßstabsdefinitionen eng mit der modernen Gesellschaft verbunden sind. Weiter erweist sich, dass auch pädagogische Theorien mit Anspruch auf Allgemeingültigkeit von Normalitätsvorstellungen geprägt sind und dass sie selbst normalisierende Wirkungen haben. Die Brüchigkeit des Anspruchs auf allgemeine Gültigkeit sowie der normalisierende Charakter verdeutlichen die versteckten Wirkungen bestimmter Subdisziplinbildungen. Das Beispiel der Interkulturellen Erziehungswissenschaft zeigt den zwiespältigen Charakter wissenschaftlicher Erklärungsansätze in seinen normierenden bzw. normalisierenden Folgen auf.

Normalisierungsprozesse in der Erziehungswissenschaft und durch die Erziehungswissenschaft können sozial wirksame Differenzen erzeugen sowie vorhandene Differenzen vergrößern. Die Wirkung solcher Normalisierungsmuster auf das und in dem Bildungswesen darf nicht unterschätzt werden. Sie ist auf allen Ebenen zu beobachten und entzieht sich durch ihre Normalität - im doppelten Sinne - weitgehend der Aufmerksamkeit. Dieser verborgene und zugleich tief greifende Einfluss solcher Muster macht das Interessante wie das Problematische aus. Es lohnt sich, diesem nachzugehen und ihn ans Licht zu holen.

\section{Literatur}

Abels, Heinz 1993: Jugend vor der Moderne. Soziologische und psychologische Theorien des 20. Jahrhunderts. Opladen: Leske + Budrich

Arbeitsgruppe Bildungsbericht am Max-Planck-Institut für Bildungsforschung 1994: Das Bildungswesen in der Bundesrepublik Deutschland. Strukturen und Entwicklungen im Überblick. Reinbek: Rowohlt

Auernheimer, Georg 1995: Einführung in die interkulturelle Erziehung. 2. überarbeitete und ergänzte Auflage (1990). Darmstadt: Wissenschaftliche Buchgesellschaft 
Beck, Iris/Düe, Willi/Wieland, Heinz (Hg.) 1996: Behindertenpädagogische und sozialpolitische Perspektiven eines Reformkonzeptes. Heidelberg: Winter

Bommes, Michael/Radtke, Frank-Olaf 1993: Institutionalisierte Diskriminierung von Migrantenkindern. Die Herstellung ethnischer Differenz in der Schule. In: Zeitschrift für Pädagogik, 39. Jg., 1993, Heft 3, S. 483-497

Bukow, Wolf-Dietrich 1996: Feindbild: Minderheit. Zur Funktion von Ethnisierung. Opladen: Leske + Budrich

Bukow, Wolf-Dietrich/Llaryora, Roberto 1993: Mitbürger aus der Fremde. Soziogenese ethnischer Minoritäten. 2. Auflage (1988). Opladen: Leske + Budrich

Bundschuh, Konrad 1997: Integration als immer noch ungelöstes Problem bei Kindern mit speziellem Förderbedarf. In: Zeitschrift für Heilpädagogik, 48. Jg., 1997, Heft 8, S. 310-315

Diehm, Isabell/Radtke, Frank-Olaf (Hg.) 1999: Erziehung und Migration. Stuttgart, Berlin, Köln: Kohlhammer

Foucault, Michel 1972: Wahnsinn und Gesellschaft. Eine Geschichte des Wahns im Zeitalter der Vernunft. 2. Auflage. Frankfurt/M.: Suhrkamp

Foucault, Michel 1976: Überwachen und Strafen. Die Geburt des Gefängnisses. Frankfurt/M.: Suhrkamp

Foucault, Michel 1977: Sexualität und Wahrheit. Der Wille zum Wissen. Frankfurt/M.: Suhrkamp

Gille, Martina/Krüger, Winfried/de Riike, Johann/Willems, Helmut 1996: Das Verhältnis Jugendlicher und junger Erwachsener zur Politik: Normalisierung oder Krisenentwicklung? In: Aus Politik und Zeitgeschichte. Beilage zur Wochenzeitung Das Parlament, B19/96 vom 3. Mai 1996, S. 3-17

Glotz, Peter 1994: Die falsche Normalisierung. Die unmerkliche Verwandlung der Deutschen. Frankfurt/M.: Suhrkamp

Gogolin, Ingrid 1994: Der monolinguale Habitus der multilingualen Schule. Münster, New York: Waxmann

Gröschke, Dieter 2000: Das Normalisierungsprinzip: Zwischen Gerechtigkeit und gutem Leben. Eine Betrachtung aus ethischer Sicht. In: Zeitschrift für Heilpädagogik, 51. Jg., 2000, Heft 4, S. 134-140

Hansen, Georg 1994: Die nationalstaatlichen Eierschalen erziehungswissenschaftlicher Theorien. In: Luchtenberg, Sigrid/Nieke, Wolfgang (Hg.) 1994: Interkulturelle Pädagogik und Europäische Dimension. Herausforderungen für Bildungssystem und Erziehungswissenschaft. Münster, New York: Waxmann, S. 189-198

Hansen, Georg 1996: Perspektivwechsel. Eine Einführung. Münster, New York: Waxmann

Hohmann, Manfred 1989: Interkulturelle Erziehung - eine Chance für Europa? In: Hohmann, Manfred/Reich, Hans H. (Hg.) 1989: Ein Europa für Mehrheiten und Minderheiten. Diskussionen um interkulturelle Erziehung. Münster, New York: Waxmann, S. 1-32

Jarausch, Konrad H. 1995: Normalisierung oder Re-Nationalisierung? Zur Umdeutung der deutschen Vergangenheit. In: Geschichte und Gesellschaft, 21. Jg., 1995, S. 571584

Koller, Christoph 1999: Lesarten. Über das Geltendmachen von Differenzen im Forschungsprozeß. In: Zeitschrift für Erziehungswissenschaft, 2. Jg., 1999, Heft 2. S. 195-209

Kremnitz, Georg 1997: Die Durchsetzung der Nationalsprachen in Europa. Münster u. a.: Waxmann 
Krüger-Potratz, Marianne 1994: Der verlängerte Arm nationalstaatlicher Bildungspolitik: Elemente völkischer Bildungspolitik in der Weimarer Republik. In: Gogolin, Ingrid (Hg.) 1994: Das nationale Selbstverständnis der Bildung. Münster, New York: Waxmann, S. 81-102

Kudlien, F./Ritter, H. H. 1984: Normal, Normalität. In: Ritter, Joachim/Gründer, KarIfried (Hg.) 1984: Historisches Wörterbuch der Philosophie. Basel, Stuttgart: Schwabe, Band 6, Spalte 920-928

Lentz, Astrid 1994: Institutionelle Grenzen interkultureller Bildung und Erziehung. In: Informationsdienst zur Ausländerarbeit, 1994, Heft 2, S. 40-43

Lentz, Carola 1998: Die Konstruktion von Ethnizität. Eine politische Geschichte NordWest Ghanas 1870-1990. Köln: Köppe

Lenzen, Dieter 1989: Pädagogik - Erziehungswissenschaft. In: Lenzen, Dieter (Hg.) 1989: Pädagogische Grundbegriffe. 2 Bände. Reinbek: Rowohlt, S. 1105-1117

Lenzen, Dieter 1999: Orientierung Erziehungswissenschaft. Was sie kann, was sie will. Reinbek: Rowohlt

Link, Jürgen 1997: Versuch über den Normalismus. Wie Normalität produziert wird. Opladen: Westdeutscher Verlag

Lutz, Helma 1999a: Das Beispiel „Ethnisierung“. Eine Kritik der Ethnisierung zehn Jahre nach „Mitbürger aus der Fremde“. In: Bukow, Wolf-Dietrich/Ottersbach, Markus (Hg.) 1999: Die Zivilgesellschaft in der Zerreißprobe. Wie reagieren Gesellschaft und Wissenschaft auf die postmoderne Herausforderung?. Opladen: Leske + Budrich, S. 117-128

Lutz, Helma 1999b: State of the Art: Zum Stand der Interkulturellen Pädagogik. In: Tertium Comparationis, 5. Jg., 1999, Heft 2 (im Druck)

Marcuse, Herbert 1967: Der eindimensionale Mensch. Studien zur Ideologie der fortgeschrittenen Industriegesellschaft. Neuwied: Luchterhand

Müssig-Trapp, Peter 2000: Heute wie vor 50 Jahren: Schule sichert die soziale Hierarchie. In: Pädagogik, 52. Jg., 2000, Heft 4, S. 48-52

Nunner-Winkler, Gertrud 1984: Normen. In: Kerber, Harald/Schmieder, Arnold (Hg.) 1984: Handbuch Soziologie. Zur Theorie und Praxis sozialer Beziehungen. Reinbek: Rowohlt, S. 398-408

Peuckert, Rüdiger 2000: Norm, soziale. In: Schäfers, Bernhard (Hg.) 2000: Grundbegriffe der Soziologie. 6. Auflage. Opladen: Leske + Budrich, S. 255-259

Radtke, Frank-Olaf 1995: Interkulturelle Erziehung. Über die Gefahren eines pädagogisch halbierten Anti-Rassismus. In: Zeitschrift für Pädagogik, 41. Jg., 1995, Heft 6, S. 853-864

Rendtorff Barbara/Moser, Vera (Hg.) 1999: Geschlecht und Geschlechterverhältnisse in der Erziehungswissenschaft. Eine Einführung. Opladen: Leske + Budrich

Rousseau, Jean-Jacques 1762: Emil oder Über die Erziehung. Vollständige Ausgabe. In neuer deutscher Fassung besorgt von Ludwig Schmidts. 3. Auflage, 1975. Paderborn: Schöningh

Schaarschuch, Andreas 1996: Soziale Arbeit in guter Gesellschaft? Gesellschaftliche Modernisierung und die „Normalisierung“ der Sozialpädagogik. In: Zeitschrift für Pädagogik, 42. Jg., 1996, Heft 6, S. 853-868

Schäfers, Bernhard 1992: Die Grundlagen des Handelns: Sinn, Normen, Werte. In: Korte, Hermann/Schäfers, Bernhard (Hg.) 1992: Einführung in Hauptbegriffe der Soziologie. Opladen: Leske + Budrich, S. 17-34

Schiffauer, Werner 1997: Fremde in der Stadt. Zehn Essays über Kultur und Differenz. Frankfurt/M.: Suhrkamp 
Schildmann, Ulrike 1997: Integrationspädagogik und Normalisierungsprinzip - ein kritischer Vergleich. In: Zeitschrift für Heilpädagogik, 48. Jg., 1997, Heft 3, S. $90-96$

Schildmann, Ulrike 2000: Forschungsfeld Normalität. Reflexionen vor dem Hintergrund von Geschlecht und Behinderung. In: Zeitschrift für Heilpädagogik, 51. Jg., 2000, Heft 3, S. 90-94

Schoch, Bruno 1996: Die Ambivalenz der Normalisierung. In: Blätter für deutsche und internationale Politik, 41. Jg., 1996, Heft 7, S. 804-813

Sierk, Udo 1995: NORMalisierung von rechts. Biopolitik und »Neue Rechte«. Hamburg: Libertäre Assoziation

Steiner-Khamsi, Gita 1996: Transnationalität und kulturelle Staatsbürgerschaft. In: Auernheimer, Georg/Gstettner, Peter (Red.) 1996: Pädagogik in multikulturellen Gesellschaften. Jahrbuch für Pädagogik 1996. Frankfurt/M. u. a.: Lang, S. 263 - 284

Waldenfels, Bernhard 1998: Grenzen der Normalisierung. Studien zur Phänomenologie des Fremden 2. Frankfurt/M.: Suhrkamp

Wenning, Norbert 1993: Migration und Ethnizität in pädagogischen Theorien. Münster, New York: Waxmann

Wenning, Norbert 1996a: Die nationale Schule. Öffentliche Erziehung im Nationalstaat. Münster, New York: Waxmann

Wenning, Norbert 1996b: Migration in Deutschland. Ein Überblick. Münster, New York: Waxmann

Wenning, Norbert 1999: Vereinheitlichung und Differenzierung. $\mathrm{Zu}$ den „wirklichen“ gesellschaftlichen Funktionen des Bildungswesens im Umgang mit Gleichheit und Verschiedenheit. Opladen: Leske + Budrich 